\title{
Adaptive Thrust Vector Control during On-Orbit Servicing
}

\author{
L. Felicetti ${ }^{1}$, M. Sabatini ${ }^{2}$, A. Pisculli ${ }^{3}$, P. Gasbarri $^{4}$ and G. B. Palmerini ${ }^{5}$ \\ University of Rome "La Sapienza”, Rome, Italy
}

\begin{abstract}
On-orbit servicing missions often include a final propulsive phase where a spacecraft pushes the other one towards a different orbit. Specifically this is the case of the debris grasping mission where the chaser, after capturing the target by means of robotic arms, has to perform a de-orbit operation. The large thrust involved needs a perfect alignment with respect to the center of mass or the system composed by chaser and target, in order to avoid attitude changes. Such accurate alignment is quite difficult to achieve especially when the characteristics of the target are not perfectly known. A procedure is proposed in this paper, allowing a complete estimation of the center of mass position and of the moments of inertia of the system, starting from the data obtained by the gyros mounted on board of the spacecraft. The output is used to design a maneuver for correcting the target and chaser relative position by moving the robotic arms. Numerical simulations show the proficiency and the applicability of the estimation algorithm and of re-alignment maneuver to a selected mission scenario.
\end{abstract}

\section{Introduction}

$\mathrm{R}^{\mathrm{c}}$ OBOTIC missions involving the docking of two spacecraft quite often include a phase during which the grasping satellite pushes the target platform. This action happens either during the contact/berthing or at the release phase. The thrust can be related to the attitude dynamics of the composite of the two satellites, or to the translational dynamics of both the spacecraft, intended to re-boost the cluster or to push away a platform from the other one ${ }^{1}$. The common issue for such a kind of maneuvers is represented by the fact that their effects will depend on the mass and inertia of the platforms as well as on the location of the thrusting point(s) with respect to the centers of mass. These characteristics are poorly known or unavailable, and the designer needs to consider these uncertainties, as they will strongly affect their resulting dynamics.

A non-cooperative rendez-vous and docking maneuver, as the one involved in the grasping of the debris, is a clear example of these issues ${ }^{2}$. In fact, the mass and the geometrical characteristics of the debris and its kinematic state could be unknown. Of course, these parameters must be evaluated in advance before defining any details of the grasping mission, but this is not always possible. Another aspect that must be considered is that, after the grasping, part of target's momentum is transferred to the chaser. A sequence of attitude maneuvers could be needed to complete the operations and re-gain the desired attitude configuration ${ }^{3-5}$. To be also reminded that the final step of a grasping mission could be represented by the chaser transfer maneuver in order to move the composite set of the two platforms in a graveyard orbit or to dive them into the atmosphere for the burn out.

One of the possibilities to perform a transfer maneuver is to use on board thrusters. The correct maneuver requires an accurate orientation of the thrust. In this paper a re-orientation maneuver, involving a servicing platform attached to a spent final stage of a launcher, will be investigated.

\footnotetext{
${ }^{1}$ Postdoctoral Researcher, DIAEE Dipartimento di Ingegneria Astronautica Elettrica ed Energetica, via Salaria 851, 00138 Rome, Italy, leonard.felicetti@uniroma1.it

${ }^{2}$ Researcher, DIAEE Dipartimento di Ingegneria Astronautica Elettrica ed Energetica, via Salaria 851, 00138 Rome, Italy, marco.sabatini@uniroma1.it

${ }^{3}$ Ph.D. Student, DIMA Dipartimento di Ingegneria Meccanica ed Aerospaziale, via Eudossiana 18, 00185 Rome, Italy, andrea.pisculli@uniroma1.it

${ }^{4}$ Associate Professor, DIMA Dipartimento di Ingegneria Meccanica ed Aerospaziale, via Eudossiana 18, 00185 Rome, Italy, paolo.gasbarri@uniroma1.it

5 Associate Professor, DIAEE Dipartimento di Ingegneria Astronautica Elettrica ed Energetica, via Salaria 851, 00138 Rome, Italy, giovanni.palmerini@uniroma1.it, AIAA Senior Member.
}

1

American Institute of Aeronautics and Astronautics 
This paper aims to analyze first the estimation of the inertia parameters, such as the center of mass location and the moments of inertia of the coupled system (chaser plus target), once the chasing and grasping phases are accomplished. The problem of the in orbit estimation of these parameters has been investigated in the past ${ }^{6-11}$. In particular, Bergman et al. ${ }^{6}$ developed a filter to extract the rigid body inertia parameters by neglecting the gyroscopic terms and in presence of known thrusters pulses, Wilson et al. ${ }^{7}$ realized analogous filters and batch estimators which also include the gyroscopic terms. The problem of the inclusion of gyroscopic terms on the system plant was bypassed by Tanygin and Williams ${ }^{8}$ using a relation directly connected to the kinetic energy of the system and, by Norman et al. ${ }^{9}$ using the balance of the angular moments of the system. Other approaches, which involve the use of the unscented Kalman filters, were developed by VanDyke et al. ${ }^{10}$ and by Sekhavat et al. ${ }^{11}$. The present work proposes the use of an extended Kalman filter, assuming that the whole system, composed by the chaser, the target and the grasping mechanisms, is a rigid body. The full set of the equations of motion are included in the estimation and the partial derivatives, related to the Kalman filter, are evaluated numerically. This procedure allows a complete estimation of the center of mass position and of the moments of inertia, by using only the gyros mounted on board of the chaser.

The second objective of this work is to study a re-alignment maneuver of the thrust direction with respect to the system center of mass. This maneuver is performed by using the arms mounted on the chaser that will be re-aligned to reduce the attitude deviations produced by the misalignment of the line of action of the thrust. The motion of the arms will be performed maintaining the clamping of the end effectors on the target.

The paper presents the theory and the simulation findings beginning (Section II) with the description of the overall mission scenario considered. Then the focus shifts on the mission phases requiring a specific attention with respect to analysis and modeling issues: Section III faces the problem of the estimation of dynamics parameters of the chaser and target system by means of an Extended Kalman Filter, which formulation and implementation's details for the specific application are reported. Following Section IV discusses the realignment maneuver before the large de-orbiting pulse. Section $\mathrm{V}$ is devoted to the numerical results, which are presented with respect to the estimation (this part is completed by an insightful robustness analysis) to the realignment and to the de-orbit phase. After the conclusions, the appendix shortly report some details on the specific procedure implemented to control the arms with the aim to provide the interested reader with a global sketch of the approach followed.

\section{Mission Scenario}

The sample mission exploiting the grasping and pushing phases considered in this paper refers to the removal of a launcher's exhausted upper stage performed by a service platform equipped with three robotic arms, as represented in Fig. 1.

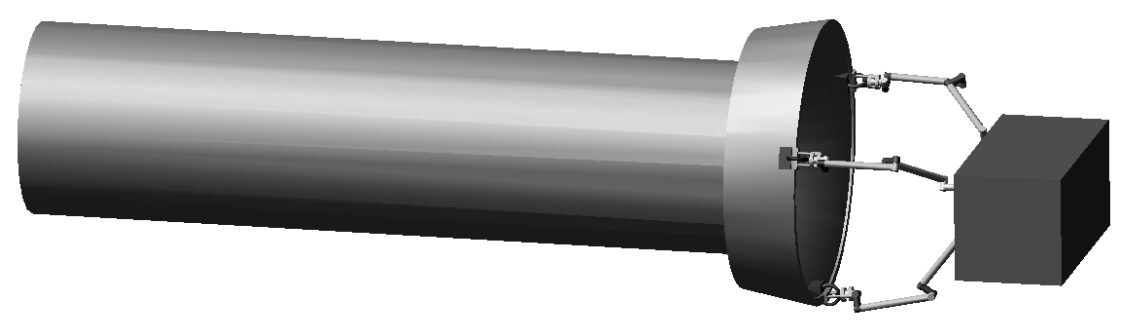

Fig. 1 Chasing spacecraft grasping an upper stage with its robotic manipulator

The overall operations can be divided in a sequence of five steps, involving different aspects and requirements in terms of guidance and control needs ${ }^{1}$ :

1) Approach and coordination: the chaser reaches a position close to the target stage and coordinates its attitude motion with respect to the one of the (non-maneuvering) stage. Only the attitude control system of the chaser and the relevant thrusters are used in this phase, while the robotic manipulators remain in their stowed configuration.

2) Arms deployment: when the desired relative kinematic state of the chaser with respect to the stage is attained, the robotic arms are deployed, bringing the end effectors in close proximity of the selected grasping zones.

3) Grasping: the robotic arms and end effector are commanded in order to catch - and firmly maintain - the stage at the selected points.

4) Post-grasping: the arms are strengthened (solidification) by blocking their joints and the de-tumbling maneuvers are performed in order to avoid any residual relative motions between the bodies and to stabilize (tranquillization) the system, which can be considered as a single, rigid body from this time on.

American Institute of Aeronautics and Astronautics 
5) De-orbit: the maneuver is performed by using a dedicated, powerful engine accommodated onboard the chaser, oriented opposite to the V-bar direction, in order to decrease the orbital altitude of the system leading to reentry.

The mission definition - and above all the grasping phase - should take into account the details of the geometry of the target. In order to allow a firm grasping, a clamping mechanism capable to perform the maneuver with respect to capture points in a thin aluminum gauge was considered. Referring to real possible targets (i.e. launcher stages' structures) a three-part tool has been designed, with two pincers to clamp the wall and a piston to block (hold) the grasp.

The arms, composed by two longer links, two joint elements (at the shoulder and at the elbow) and the end effector (the grasping mechanism outlined above) have 6 degrees of freedom each. The length of the links ensures safe operations by keeping the chaser and the target sufficiently far, as shown in Fig. 2. The adopted configuration also provides enough dexterity, needed when the target tumbling with respect to the chaser, while allowing a compact stowed configuration during the non-operational phases.

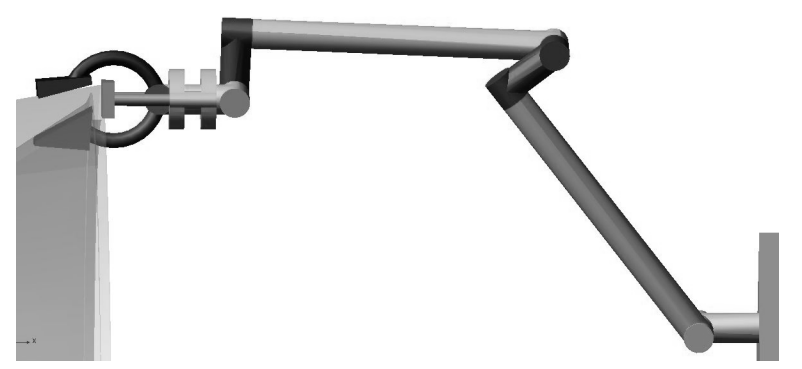

Fig. 2 The selected design of the robotic arm

\section{Estimation of the Position of the Centre of Mass and Inertia Parameters}

When the grasping phase and the following tranquillization are completed, the preparation of the large (in the order of $500 \mathrm{~N}$ ) de-orbiting thrust begins. Also with respect to possible, long duration of the de-orbit pulse, the direction of the thrust should be precisely enforced. Within this maneuver, it is easy to understand the need of an accurate estimation of the overall system center of mass and inertia. In fact, if the thrust is not perfectly aligned with the system's center of mass, the resulting torque will change the attitude of the system during the maneuver, leading to a wrong thrusting direction (Fig. 3 depicts such a possible undesired occurrence). At the same time, the values of the moments of inertia are needed to characterize the system, providing the capabilities to design eventually required reorientation maneuvers.

On the other hand, these values are practically unknown, as the characteristics of a long term orbiting body are difficult to assess. In such a concern, the case of real debris, originated by an explosion, is of course even worst with respect to a reasonably undamaged stage. Furthermore, these values are depending on the exact location of the grasping point, and on the grasping arms' configuration, so that - at best - their values can be only estimated.

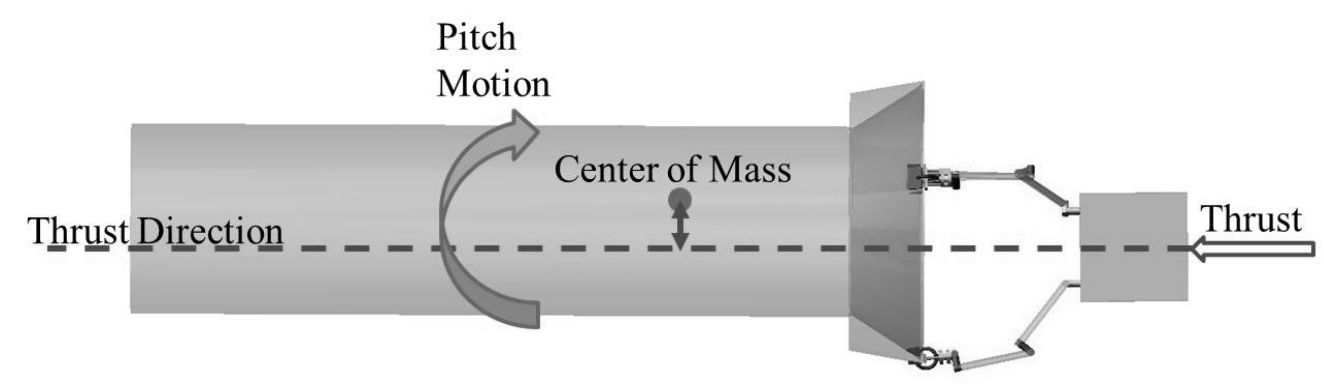

Fig. 3 Thrust misaligned with respect to the center of mass, producing a pitch motion

An estimation process, based only on the cluster of three gyros accommodated onboard the platform is therefore needed and proposed. The resulting estimates will be adopted to align the thrust to the desired direction. 
Specifically, the proposed process involves an extended Kalman filter ${ }^{12}$, which is designed on the basis of the hypothesis that the compound is solid, i.e. there is no relative motion between the chaser and the target.

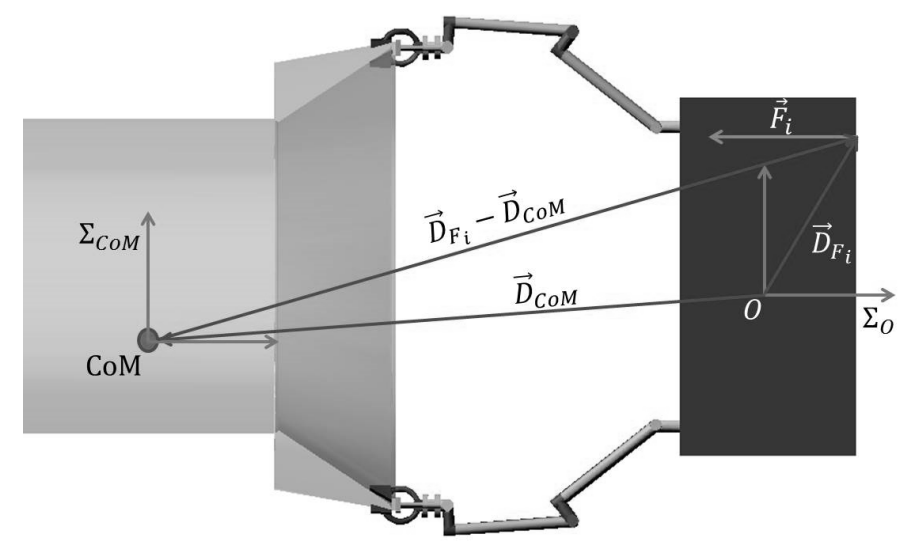

Fig. 4 Reference frames, position vectors and forces applied to the system considered as rigid body

Let us consider a rigid body system and let us associate an arbitrary reference frame $\Sigma_{O}$, centered in a point $O$ attached to the body, as depicted in Fig. 4. The position of the center of mass of the system is given by position vector $\vec{D}_{C o M}$. The position vector $\vec{D}_{F_{i}}$. represents the vector identifying the point where a thrust $\vec{F}_{i}$ is applied to the body. Considering the center of mass of the system as the pole to compute inertias and torques, the rotational dynamics reads as:

$$
\dot{\vec{\omega}}=\mathbf{I}^{-1}\left[-\vec{\omega} \times \mathbf{I} \cdot \vec{\omega}+\left(\vec{D}_{F_{i}}-\vec{D}_{C o M}\right) \times \vec{F}_{i}\right]
$$

where $\vec{\omega}$ is the angular velocity and $\mathbf{I}$ is the moment inertia matrix of the body, defined as:

$$
\mathbf{I}=\left[\begin{array}{lll}
I_{x x} & I_{x y} & I_{x z} \\
I_{y x} & I_{y y} & I_{y z} \\
I_{z x} & I_{z y} & I_{z z}
\end{array}\right]
$$

Scalar quantities $I_{x x}, I_{y y}, I_{z z}, I_{x y}=I_{y x}, I_{x z}=I_{z x}$ and $I_{y z}=I_{z y}$ represent the moments of inertia of the body referred to the axes of $\Sigma_{O}$ frame. For a rigid body the following differential relations hold:

and

$$
\begin{array}{ll}
\dot{I}_{x x}=0 & \dot{I}_{x y}=\dot{I}_{y x}=0 \\
\dot{I}_{y y}=0 & \dot{I}_{y z}=\dot{I}_{z y}=0 \\
\dot{I}_{z z}=0 & \dot{I}_{z x}=\dot{I}_{x z}=0
\end{array}
$$

$$
\dot{\vec{D}}_{C o M}=0
$$

By introducing the state vector $\mathrm{X}$ as follows:

$$
\mathrm{X}=\left[\begin{array}{llllllll}
\vec{\omega}^{T} & \vec{D}_{C o M}^{T} & I_{x x} & I_{y y} & I_{z z} & I_{x y} & I_{x z} & I_{y z}
\end{array}\right]^{T}
$$

and the control input vector as:

$$
\mathrm{U}=\left[\begin{array}{ll}
\vec{D}_{F_{i}}^{T} & \vec{F}^{T}
\end{array}\right]^{T}
$$

the equations (1), (3) and (4) can be rearranged in the following form:

$$
\dot{\mathrm{X}}=\mathrm{F}(\mathrm{X}, \mathrm{U}, t)+w
$$


where $w$ is the process noise vector, including the uncertainties of the model and the inaccuracies in the actuators' behavior. As an additional hypothesis this process noise has a mean value $E(w)=0$, while, for the covariance matrix the relation $E\left(w w^{T}\right)=\mathbf{Q}$ holds.

The angular velocities, provided by gyros mounted on board of the chaser, are the only measurements available and required for the estimation. They can be included in the filter by means of the following measurement equation:

$$
\mathrm{Z}=\mathbf{H} \mathrm{X}+\mathrm{v}
$$

where $\mathrm{Z}$ is the measurement vector and $\mathbf{H}$ is the measurement matrix:

$$
\mathbf{H}=\left[\begin{array}{lll}
\mathbf{E}_{3 \times 3} & \mathbf{0}_{3 \times 3} & \mathbf{0}_{3 \times 6}
\end{array}\right]
$$

with $\mathbf{E}$ the identity matrix, $\mathbf{0}$ the null matrix, while the indexes represent the relevant dimensions. Measurements are assumed as affected by white noise (v), with a mean value $E(v)=0$ and the covariance matrix $E\left(\mathrm{vv}^{T}\right)=\mathbf{R}$.

\section{A. Extended Kalman Filter Implementation}

At each time $\operatorname{step}\left(t_{k}=t_{k-1}+\Delta t\right)$, the filter predicts the state $\tilde{\mathrm{X}}_{k}$ by means of the $4^{\text {th }}$ order Runge-Kutta integration algorithm ${ }^{13}$, starting from the state estimate at the previous step $\hat{\mathrm{X}}_{k-1}$ :

$$
\begin{aligned}
& \mathrm{K}_{1}=\mathrm{F}\left(\hat{\mathrm{X}}_{k-1}, \mathrm{U}_{k-1}, t_{k-1}\right) \\
& \mathrm{K}_{2}=\mathrm{F}\left(\hat{\mathrm{X}}_{k-1}+\frac{\Delta t}{2} \mathrm{~K}_{1}, \mathrm{U}_{k-1}, t_{k-1}+\frac{\Delta t}{2}\right) \\
& \mathrm{K}_{3}=\mathrm{F}\left(\hat{\mathrm{X}}_{k-1}+\frac{\Delta t}{2} \mathrm{~K}_{2}, \mathrm{U}_{k-1}, t_{k-1}+\frac{\Delta t}{2}\right) \\
& \mathrm{K}_{4}=\mathrm{F}\left(\hat{\mathrm{X}}_{k-1}+\Delta t \mathrm{~K}_{3}, \mathrm{U}_{k-1}, t_{k-1}+\Delta t\right) \\
& \tilde{\mathrm{X}}_{k}=\hat{\mathrm{X}}_{k-1}+\frac{1}{6}\left(\mathrm{~K}_{1}+2 \mathrm{~K}_{2}+2 \mathrm{~K}_{3}+\mathrm{K}_{4}\right) \Delta t
\end{aligned}
$$

The prediction of the covariance matrix associated to the state is computed as follows:

$$
\tilde{\mathbf{P}}_{k}=\boldsymbol{\Phi}_{k} \hat{\mathbf{P}}_{k-1} \boldsymbol{\Phi}_{k}^{T}+\mathbf{Q}
$$

where $\boldsymbol{\Phi}_{k}$ is the transition matrix, approximated as:

$$
\mathbf{\Phi}_{k} \cong\left[\mathbf{E}_{12 \times 12}+\mathbf{J}_{12 \times 12} \Delta t\right]
$$

The Jacobian matrix $\mathbf{J}_{12 \times 12}$ is evaluated numerically, with the approximation of each element $J_{i j}$ ( $i$-th row, $j$-th column) by the following finite difference:

$$
J_{i j}=\left.\frac{\partial \mathrm{F}_{i}}{\partial \mathrm{x}_{\mathrm{j}}}\right|_{\hat{X}_{k-1}}=\frac{\mathrm{F}_{i}\left(\hat{\mathrm{X}}_{k-1}+\Delta \mathrm{X}_{j}, \hat{\mathrm{U}}, t\right)-\mathrm{F}_{i}\left(\hat{\mathrm{X}}_{i}, \hat{\mathrm{U}}, t\right)}{\Delta \mathrm{x}_{\mathrm{j}}}
$$

where $\Delta \mathrm{x}_{\mathrm{j}}$ is the increment of the $j$-th variable of the state space. The resulting $\Delta \mathrm{X}_{j}$ is the associate incremental vector, with all components void except that the $j$-th one, like:

$$
\Delta \mathrm{X}_{j}=\left[\begin{array}{lllllll}
0 & \cdots & 0 & \Delta \mathrm{x}_{\mathrm{j}} & 0 & \cdots & 0
\end{array}\right]^{T}
$$

The following estimate update reads as:

$$
\hat{X}_{k}=\tilde{X}_{k}+\mathbf{K}_{k}\left(Z_{k}-\mathbf{H} \tilde{X}_{k}\right)
$$

where $\mathbf{K}_{k}$ is the Kalman gain computed at the relevant step:

$$
\mathbf{K}_{k}=\tilde{\mathbf{P}}_{k} \mathbf{H}\left(\mathbf{H} \tilde{\mathbf{P}}_{k} \mathbf{H}^{T}+\mathbf{R}_{k}\right)^{-1}
$$

and the update of the covariance matrix is given by:

$$
\hat{\mathbf{P}}_{k}=\left(\mathbf{E}_{12 \times 12}-\mathbf{K}_{k} \mathbf{H}\right) \tilde{\mathbf{P}}_{k}
$$

$$
5
$$

American Institute of Aeronautics and Astronautics 


\section{B. Application of the Filter to the Selected Scenario}

The procedure outlined allows an estimation of all the state space variables: angular velocity $\vec{\omega}$, position of the center of the mass $\vec{D}_{C o M}$, and inertia matrix $\mathbf{I}$ of the system composed by the chaser, with the robotic arms in the extended configuration, and the target.

To apply the filter there is the need to define a unique reference frame, and to assume some initial values for the involved quantities. Let assume the center $O$ of the reference frame $\Sigma_{O}$ located in the geometrical center of the chaser bus, as shown in Fig. 4. The servicing platform holds the upper stage by means of three end effectors in three different points that, due to the uncertainness related to the previous grasping maneuvers, should not be located symmetrically. As a result of the grasping maneuver, a misalignment of the target with respect to the chaser is likely to occur.

With respect to the coarse, initial values of the variables it is reasonable to assume that the contribution related to the chaser will be known far better than the ones relevant to the target. The cylinder-like shape assumed for the latter allows to assume that its center of mass has to be close to its axis of symmetry, about half of the length, and that the two transverse moments of inertias must be higher than the axial one, leading for the target inertia matrix to

$$
\hat{\mathbf{I}}_{T}^{0}=\hat{m}_{T}^{0}\left[\begin{array}{ccc}
R_{T}^{2} / 2 & 0 & 0 \\
0 & L_{T}^{2} / 12 & 0 \\
0 & 0 & L_{T}^{2} / 12
\end{array}\right]
$$

where $R_{T}$ and $L_{T}$ are the radius and the length of the upper-stage.

The initial estimation of the system center of mass position can be obtained as:

$$
\hat{\vec{D}}_{C o M}^{0}=\frac{\hat{m}_{T}^{0} \hat{\vec{D}}_{C O M-T}^{0}+\hat{m}_{C}^{0} \hat{\vec{D}}_{C o M-C}^{0}}{\hat{m}_{T}+\hat{m}_{C}}
$$

where $\hat{\vec{D}}_{C o M-C}^{0}$ and $\hat{\vec{D}}_{C o M-T}^{0}$ are the initial estimate positions of the center of mass of the chaser $\hat{m}_{C}^{0}$ and of the target $\hat{m}_{T}^{0}$.

The initial estimate of the inertia matrix can be given by applying the Huygens-Steiner theorem ${ }^{14}$ in order to report all the inertias to the system center of mass pole, as follows:

$$
\begin{aligned}
& \hat{\mathbf{I}}^{0}=\hat{\mathbf{I}}_{T}^{0}+\hat{m}_{T}^{0}\left[\left(\hat{\vec{D}}_{C o M}^{0}-\hat{\vec{D}}_{C o M-T}^{0}\right) \times\right]\left[\left(\hat{\vec{D}}_{C o M}^{0}-\hat{\vec{D}}_{C o M-T}^{0}\right) \times\right]^{T}+ \\
& +\hat{\mathbf{I}}_{C}^{0}+\hat{m}_{C}^{0}\left[\left(\hat{\vec{D}}_{C o M}^{0}-\hat{\vec{D}}_{C o M-C}^{0}\right) \times\right]\left[\left(\hat{\vec{D}}_{C o M}^{0}-\hat{\vec{D}}_{C o M-C}^{0}\right) \times\right]^{T}
\end{aligned}
$$

where the notation $[(\vec{D}) \times]$ is used for indicating the skew symmetric matrix form of the vector $\vec{D}$ which has the following expression:

$$
[(\vec{D}) \times]=\left[\begin{array}{ccc}
0 & -D_{z} & D_{y} \\
D_{z} & 0 & -D_{x} \\
-D_{y} & D_{x} & 0
\end{array}\right]
$$

It is worth to notice that Eqs. (19) and (20) do not include any parameters of the manipulator, as it has been assumed that the mass of the robotic arms are negligible with respect to the masses of the two main bodies.

The estimation can be obtained by observing the dynamical behavior of the system to a sequence of small pulses provided by the attitude control system of the chaser. As indicated by Eq.(1), each pulse allows to estimate only the components of the center of mass position vector normal to the trust direction. So, in order to have a fully 3D reconstruction of the inertia properties of the system it is necessary to have a sequence of thrust pulses acting along different directions and applied to different points of the system(T1 to T6 in Fig. 5). 


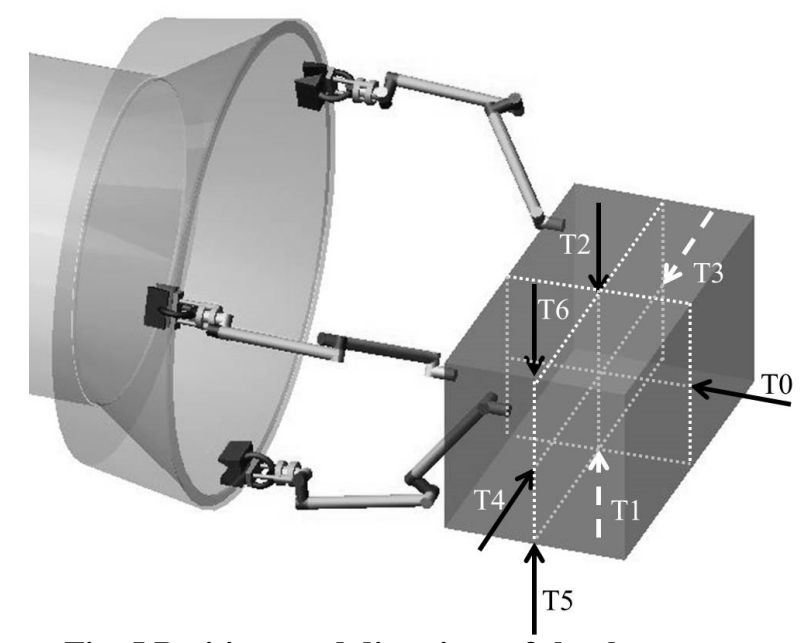

Fig. 5 Positions and directions of the thrusters

\section{Realignment Maneuver}

Once the estimates of the inertia matrix $\hat{\mathbf{I}}^{f}$ and of the position of the system center of mass $\hat{\vec{D}}_{\text {Cом }}^{f}$ are available, it is possible to correct the configuration of the system. This alignment maneuver, aimed to have the large T0 de-orbit thrust along a direction which passes through the center of mass of the system, is performed by means of the robotic arms.

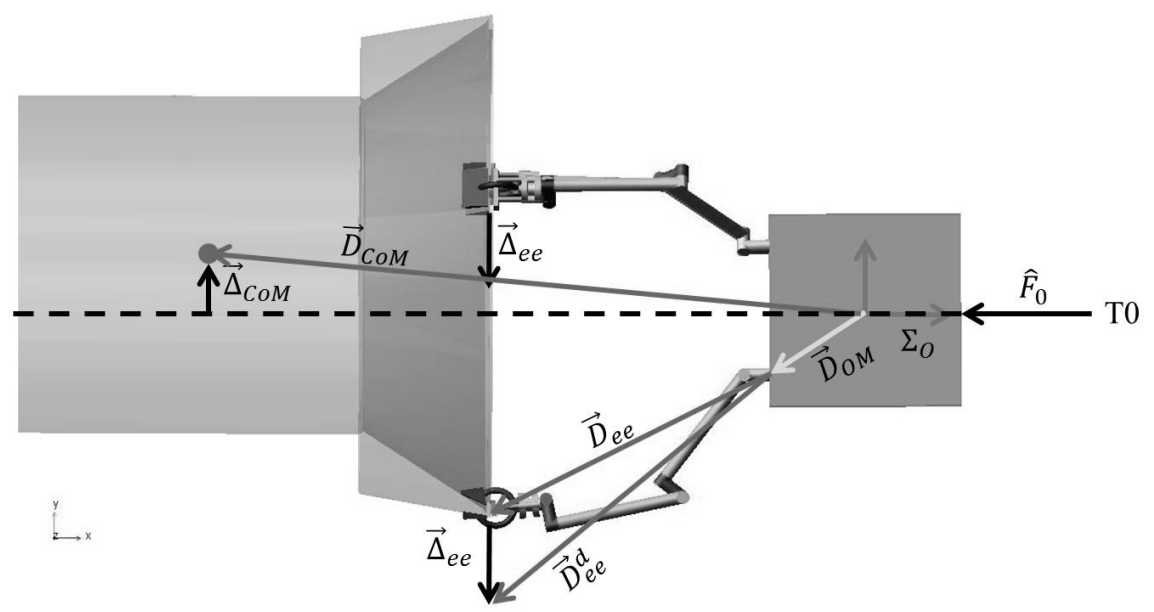

Fig. 6 Thrust alignment maneuver by means of a reconfiguration of the robotic arms

The arms are commanded to attain a desired position of their end-effectors

$$
\vec{D}_{e e}^{d}=\vec{D}_{e e}^{0}+\vec{\Delta}_{e e}
$$

starting from their initial position $\vec{D}_{e e}^{0}$, maintained along the estimation process. The term $\vec{\Delta}_{e e}$ is evaluated as the one that produces the "relative motion" between the chaser and the stage able to compensate the misalignment of the system center of mass with respect to the T0 thrust direction (axis $\hat{F}_{0}$, see Fig. 6). Such a misalignment, having only components normal to $\hat{F}_{0}$, is given by:

$$
\vec{\Delta}_{C o M}=\vec{D}_{C o M}-\left(\hat{F}_{0} \cdot \vec{D}_{C o M}\right) \hat{F}_{0}^{T}
$$


and $\vec{\Delta}_{e e}$ can be computed as:

$$
\vec{\Delta}_{e e} \cong-\frac{m_{T}}{m_{T}+m_{C}} \vec{\Delta}_{C o M}
$$

where the sign reflects the fact that - as in the Fig. 6 example - the arms shall push down to move up the chaser.

The inverse control problem can be effectively solved by means of the Jacobian transpose approach ${ }^{15}$. For each manipulator the control takes as input the relative motion of the end effector with respect to the base of the manipulator $\left(\dot{\vec{D}}_{e e}\right)$ and the difference between its current position and the desired one. These contributions, with purposely selected weighting matrices $\mathbf{K}_{p}$ and $\mathbf{K}_{d}$ are then projected along the manipulator's joints space by means of the Jacobian matrix associated $\mathbf{J}$, in order to obtain the control torques $\mathbf{C}$ commanded to the joints actuators:

$$
\mathrm{C}=\mathbf{J}^{T}\left[-\mathbf{K}_{p}\left(\vec{D}_{e e}-\vec{D}_{e e}^{d}\right)-\mathbf{K}_{d} \dot{\vec{D}}_{e e}\right]
$$

The Jacobian matrix of a manipulator can be computed on the basis of the characteristics of the links and joints. A suitable procedure exploiting the Denavit-Hartenberg ${ }^{16}$ homogenous matrices associated to each link of the multibody is reported in the Appendix.

\section{Numerical Results}

The implemented numerical simulations refer to a system (see previous Fig. 1) given by a chaser modeled as $1.5 m \times 1.5 m \times 3 m$ bus equipped with three robotic arms, and a target of cylindrical shape (10m length and $4 m$ diameter). Table 1 reports mass and inertia of the two bodies. The characteristics of the robotic arms (represented in Fig. 2) are listed in Table 2, together with the Denavit-Hartenberg parameters adopted to represent their geometrical configurations.

Table 1 Masses and moments of inertia of the chaser and of the target

\begin{tabular}{|c|c|c|c|c|c|}
\hline \multicolumn{3}{|c|}{ Chaser } & \multicolumn{3}{c|}{ Target } \\
\hline \hline \multicolumn{3}{|c|}{ Mass $(\mathrm{kg})$} & \multicolumn{3}{c|}{2500} \\
\hline \multicolumn{3}{|c|}{1000} & \multicolumn{3}{c|}{ Moments of Inertia $\left(\mathrm{kg} \cdot \mathrm{m}^{2}\right)$} \\
\hline \hline \multicolumn{2}{|c|}{ Moments of Inertia $\left(\mathrm{kg} \cdot \mathrm{m}^{2}\right)$} & \multicolumn{1}{|c|}{$I_{x x}$} & $I_{y y}$ & $I_{z z}$ \\
\hline$I_{x x}$ & $I_{y y}$ & $I_{z z}$ & 3000 & 28000 & 28000 \\
\hline 1500 & 1500 & 500 & $I_{x y}$ & $I_{x z}$ & $I_{y z}$ \\
\hline$I_{x y}$ & $I_{x z}$ & $I_{y z}$ & $I_{x y}$ & 0.0 & 0.0 \\
\hline 0.0 & 0.0 & 0.0 & 0.0 & 0.0 \\
\hline
\end{tabular}

Table 2 Denavit-Hartemberg parameters for the $\mathbf{m}$ anipulator and mass properties of the links

\begin{tabular}{|c|c|c|c|c|c|c|c|c|}
\hline & \multicolumn{3}{|c|}{ Denavit - Hartemberg parameters } & \multicolumn{5}{c|}{ Mass properties of the links } \\
\hline No. & $a_{i}(\mathrm{~m})$ & $\alpha_{i}(\mathrm{deg})$ & $d_{i}(\mathrm{~m})$ & $\vartheta_{i}(\mathrm{deg})$ & $m_{i}(\mathrm{~kg})$ & $I_{x x}\left[\mathrm{~kg} \mathrm{~m}^{2}\right]$ & $I_{y y}\left[\mathrm{~kg} \mathrm{~m}^{2}\right]$ & $I_{z z}\left[\mathrm{~kg} \mathrm{m^{2 } ]}\right.$ \\
\hline 1 & 0.0 & 90 & 0.2 & $\vartheta_{1}$ & 10.0 & 0.075 & 0.075 & 0.013 \\
\hline 2 & 0.0 & 90 & 0.1 & $\vartheta_{2}$ & 30.0 & 2.500 & 2.5 & 0.038 \\
\hline 3 & 0.2 & -90 & 1.05 & $\vartheta_{3}$ & 10.0 & 0.075 & 0.075 & 0.013 \\
\hline 4 & 0.0 & 90 & 0.1 & $\vartheta_{4}$ & 30.0 & 2.5 & 2.5 & 0.038 \\
\hline 5 & 0.2 & -90 & 1.05 & $\vartheta_{5}$ & 10.0 & 0.075 & 0.075 & 0.013 \\
\hline 6 & 0.0 & 90 & 0.1 & $\vartheta_{6}$ & 10.0 & 0.075 & 0.075 & 0.013 \\
\hline
\end{tabular}

The large de-orbit thrust is provided by a single motor (position T0 in Fig. 5) while the small pulses used for the estimation procedure are given by attitude correction thrusters (located in T1-T6). Table 3 reports the position, the thrusting direction and the magnitude of the pulses adopted in the simulation for all these components (these 8

American Institute of Aeronautics and Astronautics 
characteristics are considered perfectly known and ideally implemented). To notice that the magnitude of the small attitude correction pulses, even if limited to save propellant, must be high enough to produce effective gyro's measurements. The sequence of thruster fires has been chosen in order to have torques on orthogonal directions with respect to the body axis, with a duration of $\Delta t_{b}=150 \mathrm{~s}$.

Table 3 Location, action direction and module of the thrusters

\begin{tabular}{|c|c|c|c|c|}
\hline Thruster & Position $(m)$ & Direction & Module $(N)$ \\
\hline T0 & {$\left[\begin{array}{lll}+0.75 & 0 & 0\end{array}\right]$} & {$\left[\begin{array}{lll}-1 & 0 & 0\end{array}\right]$} & 500 \\
\hline T1 & {$\left[\begin{array}{lll}0 & -0.75 & 0\end{array}\right]$} & {$\left[\begin{array}{lll}0 & +1 & 0\end{array}\right]$} & 0.5 \\
\hline T2 & {$\left[\begin{array}{lll}0 & +0.75 & 0\end{array}\right]$} & {$\left[\begin{array}{lll}0 & -1 & 0\end{array}\right]$} & 0.5 \\
\hline T3 & {$\left[\begin{array}{lll}0 & 0 & -1.50\end{array}\right]$} & {$\left[\begin{array}{lll}0 & 0 & +1\end{array}\right]$} & 0.5 \\
\hline T4 & {$\left[\begin{array}{lll}0 & 0 & +1.50\end{array}\right]$} & {$\left[\begin{array}{lll}0 & 0 & -1\end{array}\right]$} & 0.5 \\
\hline T5 & {$\left[\begin{array}{lll}0 & -0.75 & +1.5\end{array}\right]$} & {$\left[\begin{array}{lll}0 & +1 & 0\end{array}\right]$} & 0.5 \\
\hline T6 & {$\left[\begin{array}{lll}0 & -0.75 & +1.5\end{array}\right]$} & {$\left[\begin{array}{lll}0 & -1 & 0\end{array}\right]$} & 0.5 \\
\hline
\end{tabular}

The measurement of the angular velocity of the system is performed by gyroscopes mounted on board of the chaser. The measurements are affected by white noise with a standard deviation $\sigma_{\omega}=10^{-3} \mathrm{rad} / \mathrm{s}$, with a sampling time $\Delta t_{s}=0.05 \mathrm{~s}$ on each of the three axes.

\section{A. Center of Mass Estimations Results}

The estimation procedure outlined in Sec. III, is based on the application of the sequence of thruster fires followed by an additional no-thrust phase of $\Delta t_{c}=300 \mathrm{~s}$, as shown in Fig. 7. The sequence is repeated for three times, in order to improve the convergence of the estimation. In each phase, the filter continues to estimate the extended state space by taking the last estimation results obtained in the previous phase.

After a trial and error process, the covariance matrix of the process noise has been selected as:

$$
\mathbf{Q}=\left[\begin{array}{ccc}
10^{-12} \mathbf{E}_{3 \times 3} & 0 & 0 \\
0 & 10^{-16} \mathbf{E}_{3 \times 3} & 0 \\
0 & 0 & 10^{-16} \mathbf{E}_{6 \times 6}
\end{array}\right]
$$

while the covariance matrix of the measurement noise, taking into account the characteristics of the adopted gyros, reads as:

$$
\mathbf{R}=10^{-6} \mathbf{E}_{3 \times 3}
$$

The filter is initialized by the following initial guess:

$$
\begin{aligned}
\hat{\vec{\omega}}^{0}=\left[\begin{array}{lll}
0 & 0 & 0
\end{array}\right]^{T} \mathrm{rad} / \mathrm{s} & \hat{\vec{D}}_{\text {CoM }}^{0}=\left[\begin{array}{lll}
-3 & 0 & 0
\end{array}\right]^{T} \mathrm{~m} \\
\hat{\mathbf{I}}^{0} & =\left[\begin{array}{ccc}
5 \cdot 10^{3} & 0 & 0 \\
0 & 1.1 \cdot 10^{5} & 0 \\
0 & 0 & 1.1 \cdot 10^{5}
\end{array}\right] \mathrm{kg} \cdot \mathrm{m}^{2}
\end{aligned}
$$

with an initial covariance matrix like:

where:

$$
\hat{\mathbf{P}}^{0}=\left[\begin{array}{ccc}
\hat{\mathbf{P}}_{\bar{\omega}}^{0} & 0 & 0 \\
0 & \hat{\mathbf{P}}_{C o M}^{0} & 0 \\
0 & 0 & \hat{\mathbf{P}}_{\mathbf{I}}^{0}
\end{array}\right]
$$

$$
9
$$

American Institute of Aeronautics and Astronautics 


$$
\begin{gathered}
\hat{\mathbf{P}}_{\bar{\omega}}^{0}=10^{-2} \mathbf{E}_{3 \times 3} \quad \hat{\mathbf{P}}_{C o M}^{0}=\mathbf{E}_{3 \times 3} \\
\hat{\mathbf{P}}_{\mathbf{I}}^{0}=\left[\begin{array}{ccc}
10^{-6} \mathbf{E}_{1 \times 1} & 0 & 0 \\
0 & 10^{-8} \mathbf{E}_{2 \times 2} & 0 \\
0 & 0 & 10^{-6} \mathbf{E}_{3 \times 3}
\end{array}\right]
\end{gathered}
$$
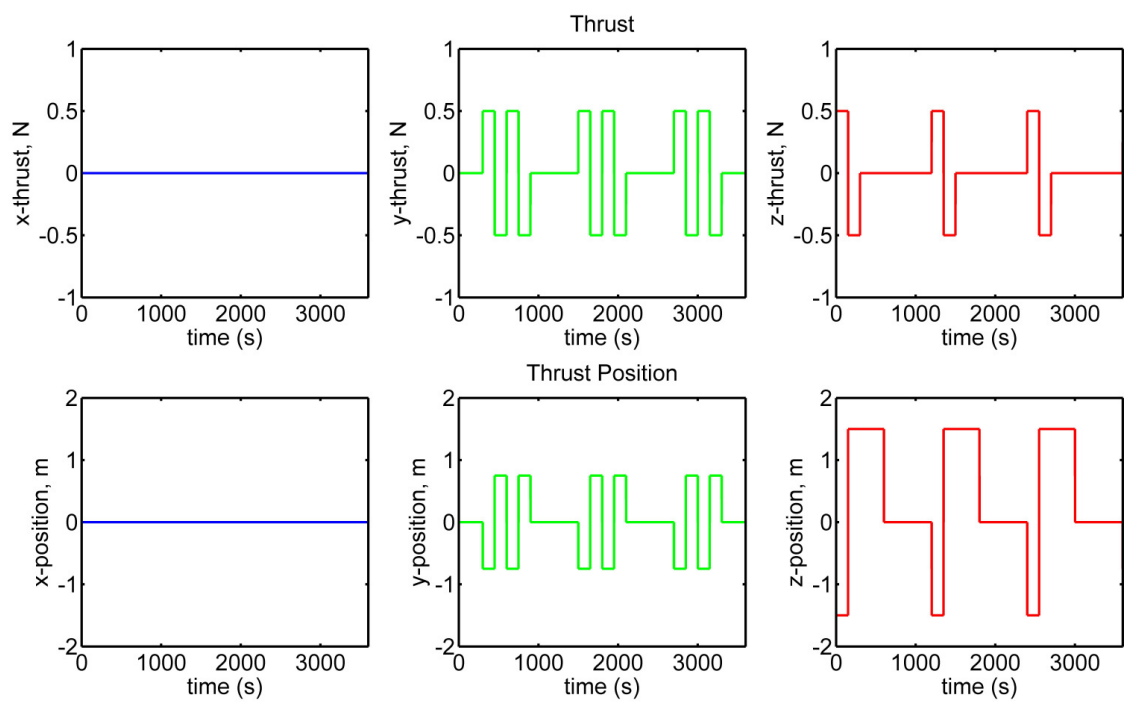

Fig. 7 Sequence of the thrust pulses and their application points on the chaser bus

The results of the estimation algorithm are reported in the following figures. In particular the angular rates measured by the gyroscopes and the estimated angular velocity of the system during the maneuver are shown in Fig. 8. The Kalman filter identifies all the changes in the attitude motion of the platform. The attitude of the system during the estimation maneuver is represented in Fig. 9.

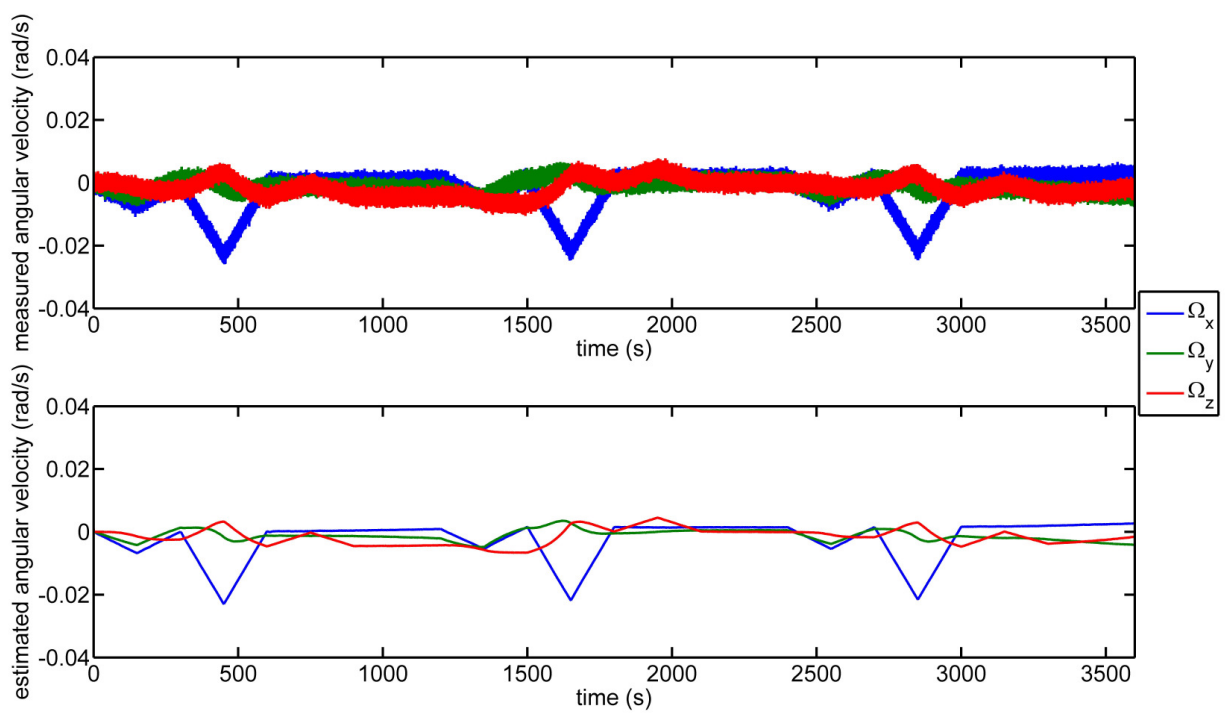

Fig. 8 Measured and estimated angular velocity during the thrust sequences operations

American Institute of Aeronautics and Astronautics 

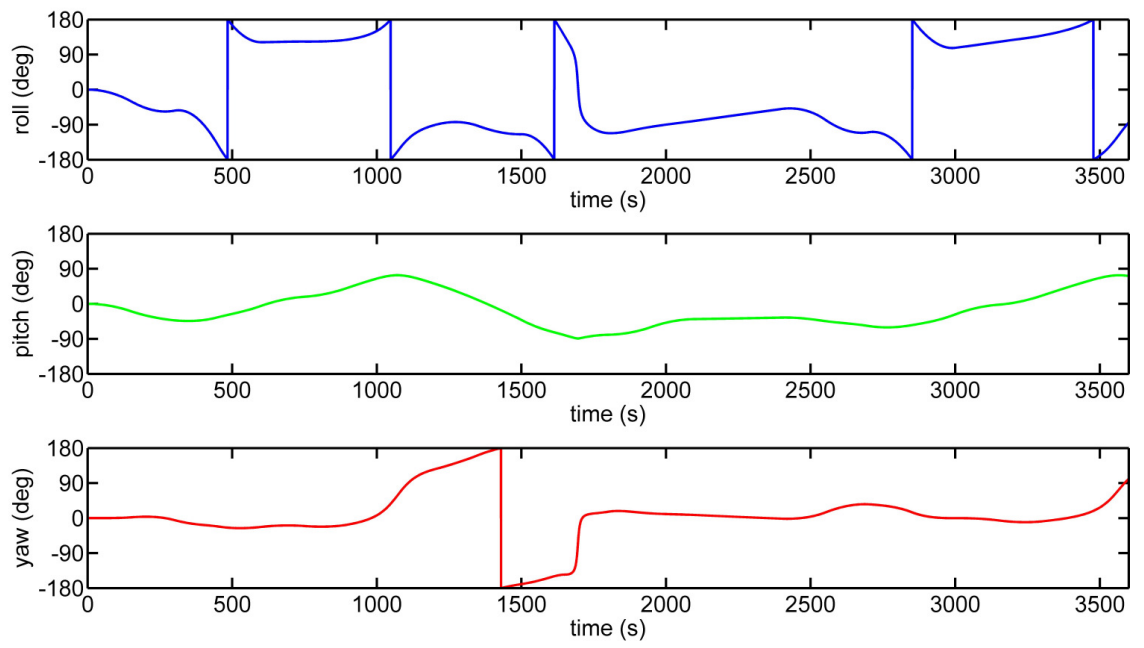

Fig. 9 Euler angles representing the system's attitude during the thrust sequences operations

The results of the estimation of the position of the center of mass are shown in Fig. 10. It is worth to notice that the component along the x-axis decreases rapidly towards the correct value, represented by a faded blue line in the plot. The same behavior is evident looking at the trend of the standard deviation of the associated error, which decreases until it reaches a steady value.

The estimation of the z-component of the center of mass position follows a different behavior as it remains constant during the first $300 \mathrm{~s}$. This initial trend is due to the fact that the thrust is first applied along the $\mathrm{z}$-axis of the chaser reference frame, leading to the impossibility of an estimation of the component along this direction (see the cross product in Eq.(1)). After $t=300 s$, the thrust direction changes and the filter actually begins to evaluate also this component of the state. Similarly, the standard deviation of the error on the z-direction remains constant to its initial value to quickly decrease towards a steady value when thrust pulses act along a different direction.

The results of the estimation of the moments of inertia are plotted in Fig. 11. With respect to the previous evaluation of the center of mass, this process clearly shows a slower convergence. During the first phases of the thrust sequence, the estimation of the moment of inertia about the x-axis is not correct. When a roll torque is applied to the system, by means of the activation of the T5 and T6 thrusters (which are misaligned with respect to the roll axis), the estimation of that moment of inertia occurs. In fact the covariance related to this moment of inertia begins to decrease rapidly after $t=600 \mathrm{~s}$. The estimation of the other moments of inertia takes a longer time to converge towards the correct values, which are reported in Table 4 for the specific case of this simulation. The associated standard deviations decrease with steps that occur at every change of the thrust directions, remarking the importance of the switching among the thrusters.

Table 4 True center of mass position and moments of inertia of the whole system

\begin{tabular}{|c|c|c|c|c|c|}
\hline \multicolumn{6}{|c|}{ Center of Mass Position (m) } \\
\hline \multicolumn{2}{|c|}{$P_{x}$} & \multicolumn{2}{|c|}{$P_{y}$} & \multicolumn{2}{|c|}{$P_{z}$} \\
\hline \multicolumn{2}{|c|}{-6.452} & \multicolumn{2}{|c|}{0.284} & \multicolumn{2}{|c|}{0.014} \\
\hline \multicolumn{6}{|c|}{ Moments of Inertia $\left(\mathrm{kg} \cdot \mathrm{m}^{2}\right)$} \\
\hline$I_{x x}$ & $I_{y y}$ & $I_{z z}$ & $I_{x y}$ & $I_{x z}$ & $I_{y z}$ \\
\hline 4951.8 & 112272.3 & 111232.5 & -2862.9 & 305.7 & 39.1 \\
\hline
\end{tabular}



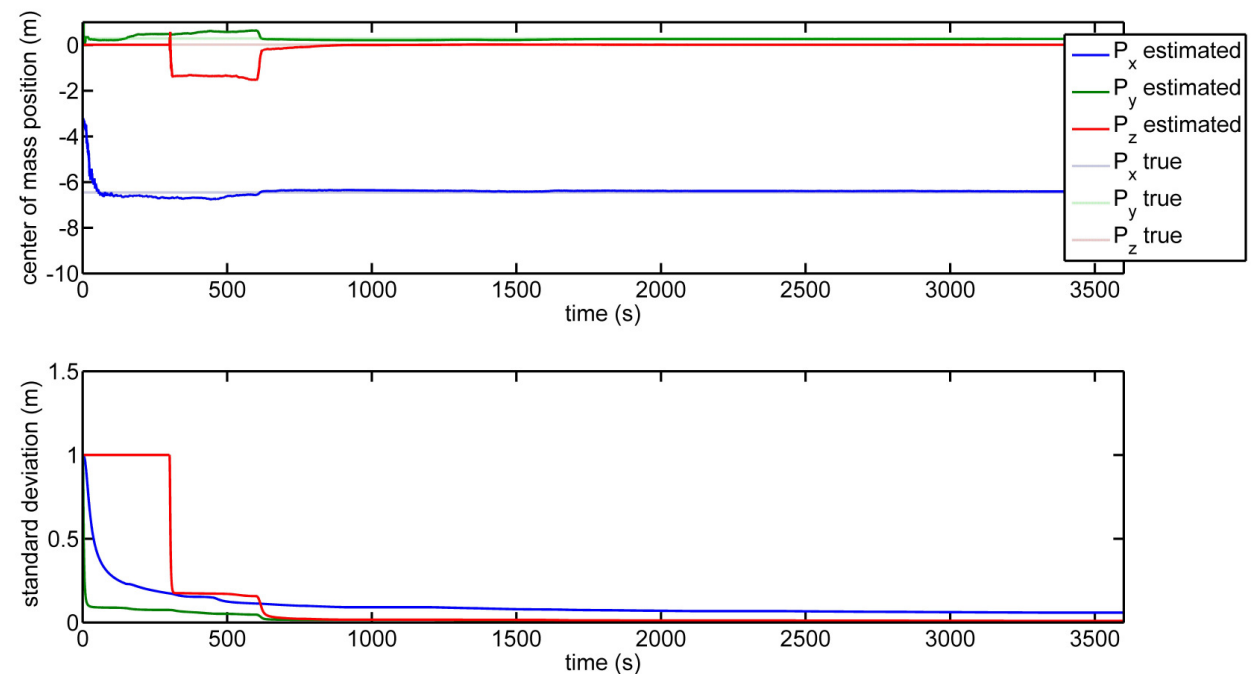

Fig. 10 Center of mass estimation and related standard deviation
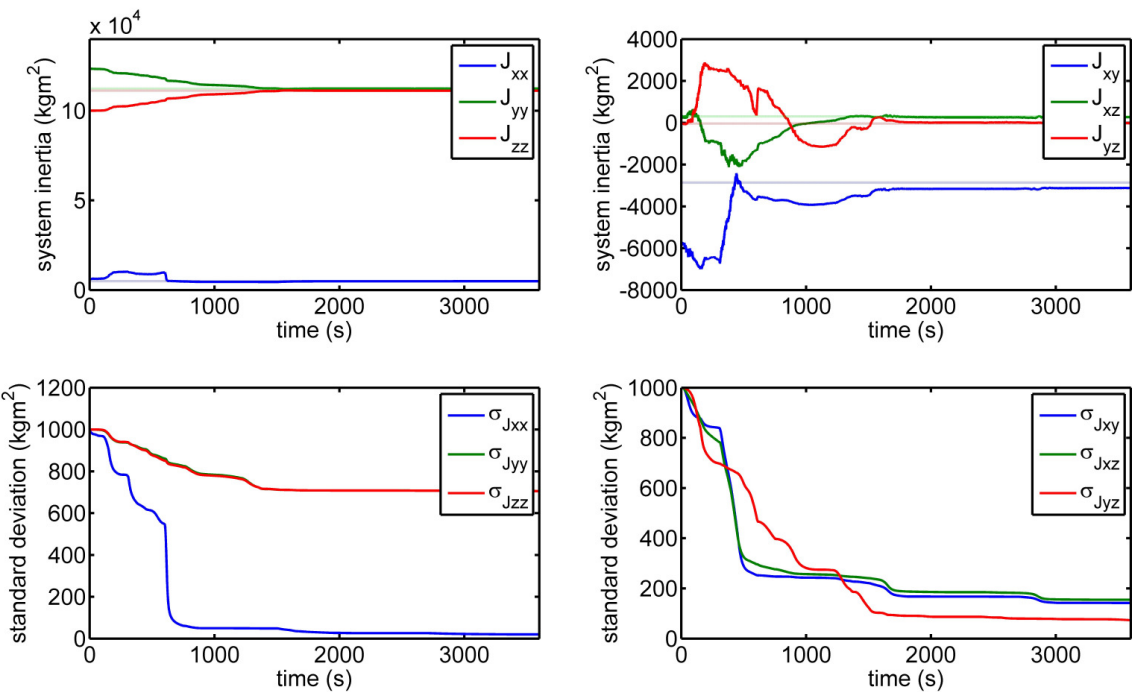

Fig. 11 Estimated moments of inertia and related standard deviations

\section{B. Estimation Robustness}

The previous results have been obtained by assuming the availability of a reasonable initial guess of the center of mass position and of the inertia properties of the system that is not always true. Moreover, due to the intrinsically non-linear nature of the system plant (see Eq.(1)), the convergence of the filter on the actual values cannot be ensured. The time required to converge, also with respect to the three-impulse strategy, is an additional aspect to consider.

A parametric study has been therefore performed in order to analyze the robustness and the convergence of the proposed extended Kalman filter. That analysis takes into account different center of mass positions, both along the axial direction of the system (x-axis) and along the two transversal directions (y-axis and z-axis) with respect to the $\Sigma_{o}$ reference frame. The findings of this parametric analysis are reported in Table 5 and Table 6, with the process starting as before from (wrong) initial guesses in Eq.(28) and, after a thrust sequence, producing as output the updated estimates for the center of mass position $\hat{\vec{D}}_{C o M}^{f}$ and the moments of inertia of the system $\hat{\mathbf{I}}^{f}$. 
Table 5 lists, with respect to the initial error on the effective center of mass location the differences between its final estimate and its true position $\left(\vec{e}_{D}=\hat{\vec{D}}_{C o M}^{f}-\vec{D}_{C o M}^{f}\right)$ and the standard deviations computed by the filter: the cases that do not satisfy the condition $\left|\vec{e}_{D}\right|<3 \sigma_{D}$ are highlighted in gray. As expected, there is a limit on the initial estimate error ( $9 \mathrm{~m}$ along the axis) to have the filter working successfully. The same analysis, leading to similar findings, is reported, in Table 6, with the errors on the final estimates of the moments of inertia $\left(e_{I}=\hat{I}_{i}^{f}-I_{i}\right)$ and the relevant standard deviations. The larger errors are related to $I_{y y}$ and $I_{z z}$, also due to the fact they are the largest moments of inertia.

Table 5 Analysis of the convergence of the filter (center of mass estimation)

\begin{tabular}{|c|c|c|c|c|c|c|}
\hline$\vec{D}_{C o M}$ & $e_{D x}$ & $e_{D y}$ & $e_{D z}$ & $\sigma_{D x}$ & $\sigma_{D y}$ & $\sigma_{D z}$ \\
\hline$\left[\begin{array}{lll}0 & 0 & 0\end{array}\right]$ & -0.022 & 0.002 & -0.000 & 0.047 & 0.004 & 0.003 \\
\hline$\left[\begin{array}{lll}-3 & 0 & 0\end{array}\right]$ & -0.030 & 0.003 & -0.001 & 0.180 & 0.007 & 0.005 \\
\hline$\left[\begin{array}{lll}-6 & 0 & 0\end{array}\right]$ & 0.144 & 0.010 & -0.001 & 0.309 & 0.011 & 0.008 \\
\hline$\left[\begin{array}{lll}-9 & 0 & 0\end{array}\right]$ & 0.743 & 0.027 & -0.003 & 0.407 & 0.014 & 0.011 \\
\hline$\left[\begin{array}{lll}-12 & 0 & 0\end{array}\right]$ & 0.888 & 0.030 & 0.001 & 0.265 & 0.012 & 0.010 \\
\hline$\left[\begin{array}{lll}-15 & 0 & 0\end{array}\right]$ & 1.284 & 0.039 & -0.000 & 0.249 & 0.011 & 0.007 \\
\hline$\left[\begin{array}{lll}-20 & 0 & 0\end{array}\right]$ & 4.601 & 0.126 & -0.017 & 0.430 & 0.019 & 0.014 \\
\hline$\left[\begin{array}{lll}-6.45 & 0.5 & 0\end{array}\right]$ & 0.177 & 0.011 & -0.002 & 0.323 & 0.011 & 0.009 \\
\hline$\left[\begin{array}{lll}-6.45 & 1.0 & 0\end{array}\right]$ & 0.137 & 0.008 & -0.002 & 0.223 & 0.009 & 0.008 \\
\hline$\left[\begin{array}{lll}-6.45 & 1.5 & 0\end{array}\right]$ & 0.045 & 0.003 & -0.002 & 0.130 & 0.008 & 0.005 \\
\hline$\left[\begin{array}{lll}-6.45 & 2.0 & 0\end{array}\right]$ & 0.085 & 0.005 & -0.002 & 0.101 & 0.008 & 0.005 \\
\hline$\left[\begin{array}{lll}-6.45 & 0 & 0.5\end{array}\right]$ & 0.210 & 0.018 & -0.002 & 0.344 & 0.015 & 0.014 \\
\hline$\left[\begin{array}{lll}-6.45 & 0 & 1.0\end{array}\right]$ & 0.218 & 0.016 & -0.002 & 0.284 & 0.014 & 0.012 \\
\hline$\left[\begin{array}{lll}-6.45 & 0 & 1.5\end{array}\right]$ & 0.265 & 0.012 & -0.004 & 0.330 & 0.011 & 0.009 \\
\hline$\left[\begin{array}{lll}-6.45 & 0 & 2.0\end{array}\right]$ & 0.182 & 0.008 & -0.004 & 0.263 & 0.008 & 0.006 \\
\hline
\end{tabular}

Table 6 Analysis of the convergence of the filter (moments of inertia)

\begin{tabular}{|c|c|c|c|c|c|c|c|c|c|c|c|c|}
\hline$\vec{D}_{C o M}$ & $e_{I x x}$ & $e_{I y y}$ & $e_{I z z}$ & $e_{I x y}$ & $e_{I x z}$ & $e_{1 y z}$ & $\sigma_{I x x}$ & $\sigma_{l y y}$ & $\sigma_{I z z}$ & $\sigma_{I x y}$ & $\sigma_{I x z}$ & $\sigma_{I y z}$ \\
\hline$\left[\begin{array}{lll}0 & 0 & 0\end{array}\right]$ & -16.4 & -8211.6 & 3720.2 & 287.7 & -27.9 & 134.4 & 18.1 & 9355.8 & 8362.9 & 269.1 & 138.9 & 995.8 \\
\hline$\left[\begin{array}{lll}-3 & 0 & 0\end{array}\right]$ & -9.7 & 274.2 & 532.5 & 72.5 & -7.8 & -18.1 & 17.6 & 6488.4 & 6489.0 & 204.6 & 144.5 & 180.3 \\
\hline$\left[\begin{array}{lll}-6 & 0 & 0\end{array}\right]$ & -9.5 & -2909.8 & -2888.9 & 148.8 & -7.1 & -7.6 & 17.5 & 5821.5 & 5822.3 & 191.0 & 144.2 & 53.4 \\
\hline$\left[\begin{array}{lll}-9 & 0 & 0\end{array}\right]$ & -12.7 & -9308.3 & -9299.8 & 303.5 & -30.8 & -2.6 & 17.5 & 5153.0 & 5154.2 & 174.9 & 134.0 & 25.7 \\
\hline$\left[\begin{array}{lll}-12 & 0 & 0\end{array}\right]$ & -10.1 & -8198.6 & -8203.3 & 241.9 & 21.4 & 2.7 & 16.9 & 2520.1 & 2521.4 & 105.0 & 90.6 & 5.8 \\
\hline$\left[\begin{array}{lll}-15 & 0 & 0\end{array}\right]$ & -10.1 & -9394.8 & -9402.5 & 264.8 & 6.0 & 0.6 & 16.9 & 1894.3 & 1894.9 & 81.2 & 51.5 & 3.2 \\
\hline$\left[\begin{array}{lll}-20 & 0 & 0\end{array}\right]$ & -24.3 & -25748.7 & -25764.6 & 688.3 & -97.3 & -2.3 & 17.1 & 2445.9 & 2447.1 & 106.7 & 78.6 & 6.5 \\
\hline$\left[\begin{array}{lll}-6.45 & 0.5 & 0\end{array}\right]$ & -6.7 & -3298.5 & -3314.6 & 143.5 & -22.7 & -1.7 & 18.0 & 5723.7 & 5725.9 & 195.7 & 152.0 & 63.1 \\
\hline$\left[\begin{array}{lll}-6.45 & 1.0 & 0\end{array}\right]$ & -2.2 & -2657.8 & -2664.6 & 78.6 & -26.4 & 1.2 & 17.7 & 4078.8 & 4080.5 & 156.9 & 123.4 & 22.9 \\
\hline$\left[\begin{array}{lll}-6.45 & 1.5 & 0\end{array}\right]$ & -0.1 & -934.5 & -933.6 & -2.5 & -12.4 & 0.2 & 17.7 & 2173.9 & 2174.9 & 81.4 & 73.9 & 3.8 \\
\hline$\left[\begin{array}{lll}-6.45 & 2.0 & 0\end{array}\right]$ & -1.6 & -1472.7 & -1474.4 & 20.8 & -27.1 & -0.7 & 17.0 & 1617.5 & 1618.1 & 66.0 & 64.4 & 3.8 \\
\hline$\left[\begin{array}{lll}-6.45 & 0 & 0.5\end{array}\right]$ & -16.5 & -3839.4 & -3869.5 & 263.2 & -30.9 & -4.3 & 18.9 & 6027.9 & 6027.9 & 260.2 & 244.6 & 74.6 \\
\hline$\left[\begin{array}{lll}-6.45 & 0 & 1.0\end{array}\right]$ & -11.2 & -3955.3 & -3972.7 & 223.7 & -28.9 & 4.0 & 19.9 & 4977.1 & 4979.4 & 238.4 & 211.2 & 21.3 \\
\hline$\left[\begin{array}{lll}-6.45 & 0 & 1.5\end{array}\right]$ & -10.7 & -4732.1 & -4745.6 & 164.0 & -45.2 & -2.9 & 17.7 & 5749.0 & 5750.6 & 191.2 & 142.0 & 33.6 \\
\hline$\left[\begin{array}{lll}-6.45 & 0 & 2.0\end{array}\right]$ & -7.7 & -3238.5 & -3237.4 & 90.0 & -35.7 & -0.0 & 16.6 & 4458.7 & 4459.8 & 135.2 & 78.3 & 20.7 \\
\hline
\end{tabular}




\section{Realignment Maneuver Results}

Once correctly estimated the position of the overall system's center of mass, the required realignment maneuver can be performed. The configuration of the manipulators before and after the realignment maneuver is sketched in Fig. 12, clearly showing the relative motion produced between the target and the chaser. The effectiveness of the simulated maneuver is presented in Fig. 13, where it is possible to notice that the initial misalignment of the center of mass is corrected in about $30 \mathrm{~s}$.

During this alignment, the end effectors do not lose their clamping point on the fairing, and the motion is obtained only by means of the changes in the links' configuration. The manipulator joints' behavior for the three robotic arms and the torques applied to the joints during the maneuver are shown in Fig. 14 and in Fig. 15, respectively.
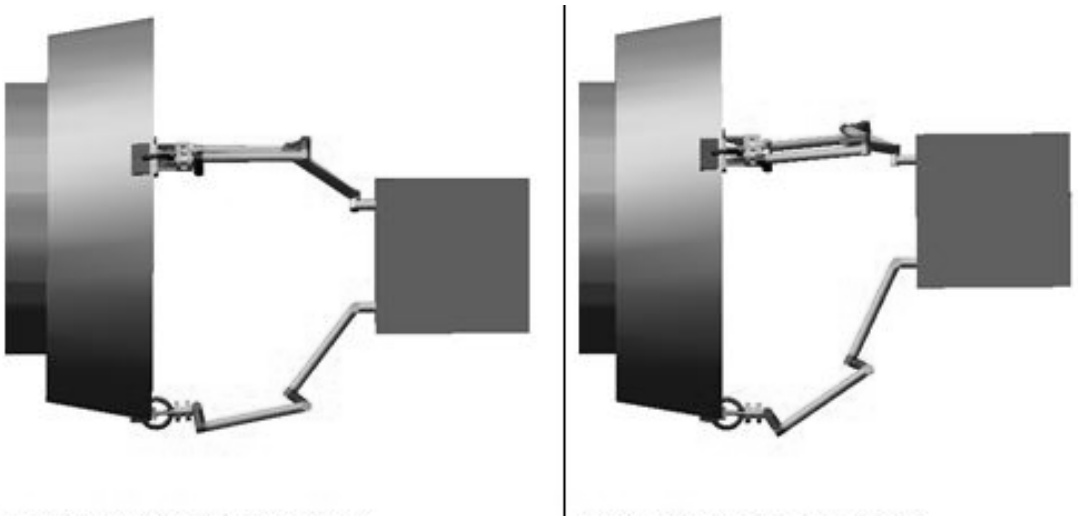

Fig. 12 System configuration before (on the left) and after (on the right) the alignment maneuver

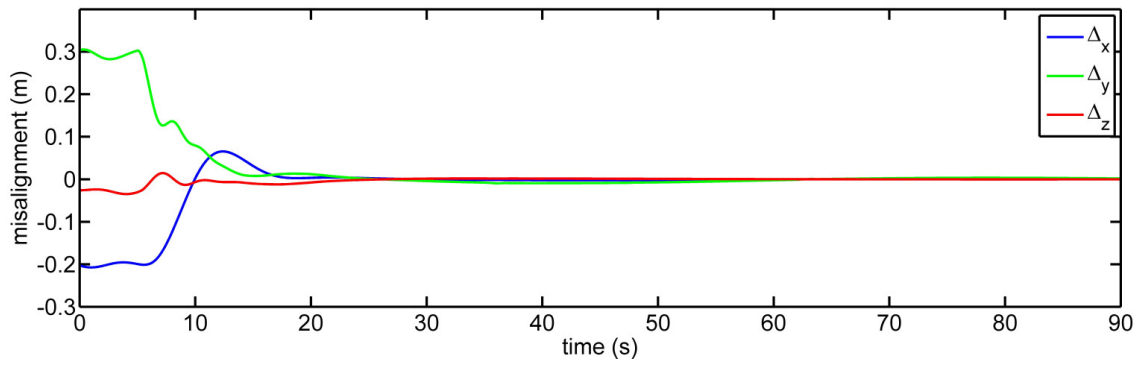

Fig. 13 Center of mass misalignment with respect to the thrust direction
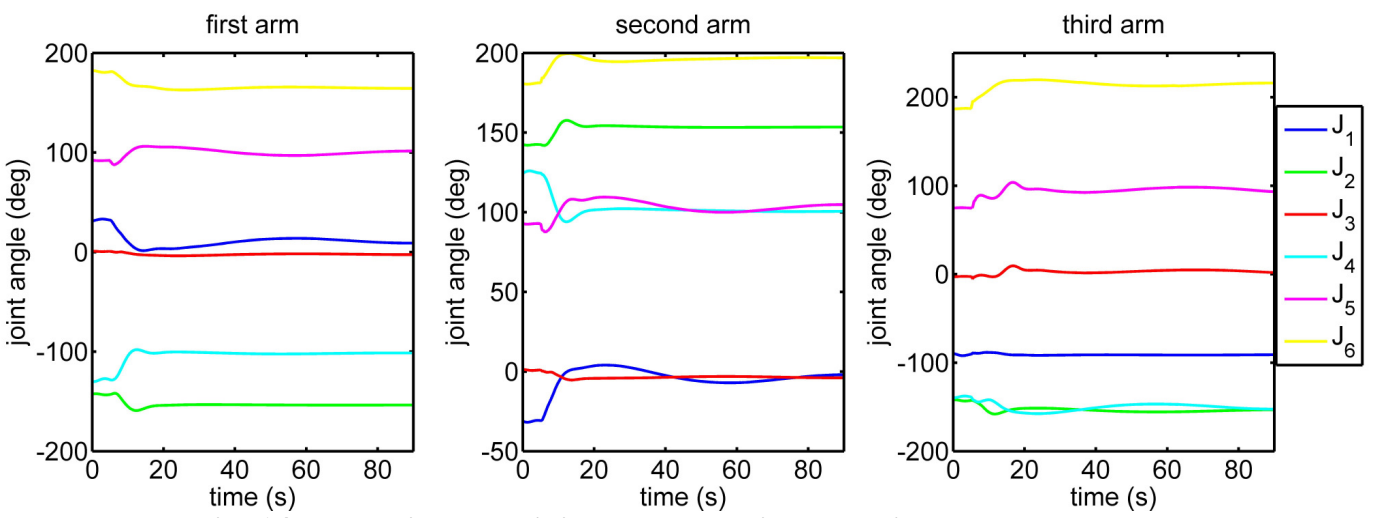

Fig. 14 Manipulator joint angles during the alignment maneuver

14

American Institute of Aeronautics and Astronautics 

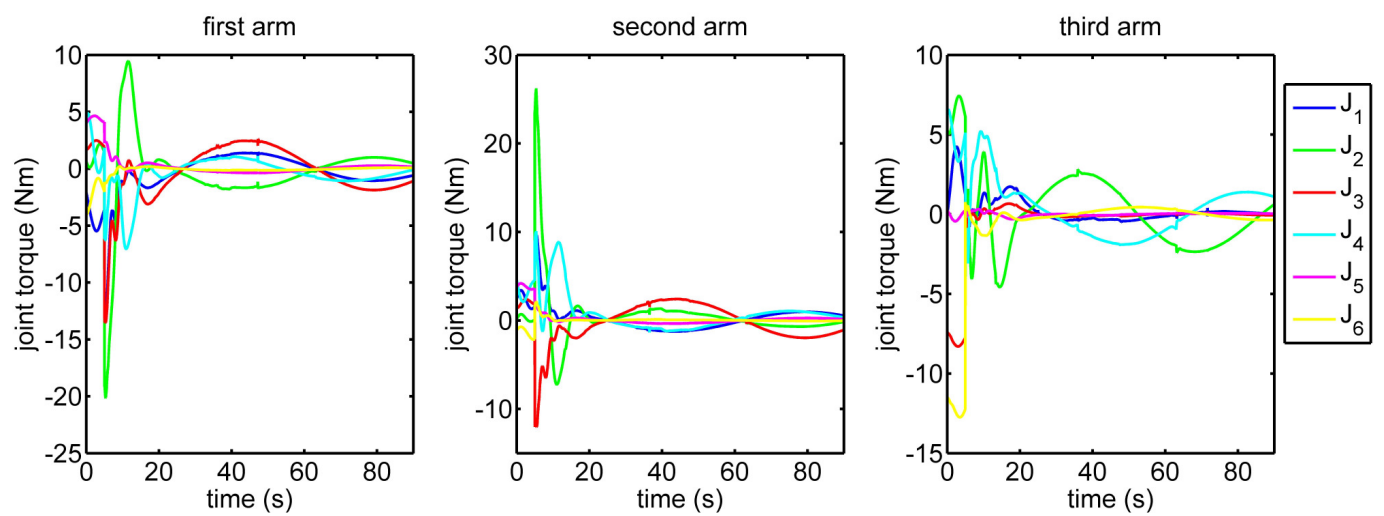

Fig. 15 Torques applied to the joints during the alignment maneuver

\section{Propulsive phase results}

Once that the stabilization, de-tumbling and the realignment maneuvers have been accomplished, the de-orbit phase can take place by means of a $500 \mathrm{~N}$ thrust applied in the T0 position in Fig. 5. To gain some insights about the effects of the misalignment of the center of mass with respect to the thrust direction, a comparative analysis has been done between aligned- and not aligned-thrusting cases. In both the cases, the reference frame associated to the system of bodies $\Sigma_{O}$ is completely aligned with the inertial reference frame and the thrust is applied at time $t_{b}=5 \mathrm{~s}$.

Fig. 16 reports the Euler angles representing the attitude of the system during the non-aligned propulsive phases. The main effect of the misalignment of the center of mass, which is located above the thrust direction

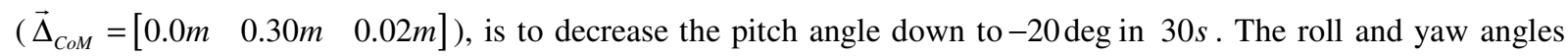
diverge with quite limited rates: during the $30 \mathrm{~s}$ of the burning phase, the roll angle increase its value up to $1.5 \mathrm{deg}$ and the yaw angle up to $2.7 \mathrm{deg}$. It is possible to suppose that these two last rates can be compensated by some limited thrust pulses along directions normal to the main thrust or by limited attitude corrections applied to the chaser. On the other hand, the larger pitch motion rate does not allow this kind of solution and the only way to solve the problem is to perform a re-alignment maneuver by means of the robotic manipulator.
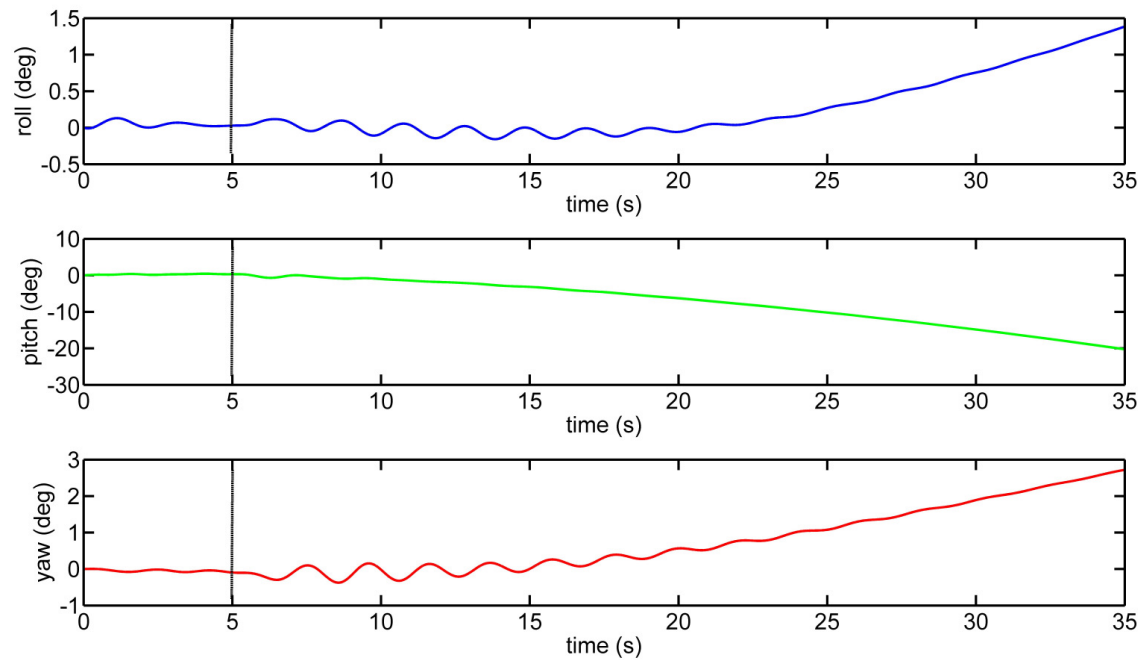

Fig. 16 Euler angles representing the system's attitude during the propulsive phase (misaligned case)

The results of the re-alignment maneuver are evident in the Euler angles behavior represented in Fig. 17. In particular the thrust, which starts at $t_{b}=5 \mathrm{~s}$, produces limited rates about all the three directions: the roll angle does 
not changes, while the pitch and yaw angles have variations of about $0.2 \mathrm{deg}$ in $30 \mathrm{~s}$, essentially due to the remaining misalignment after the maneuver.
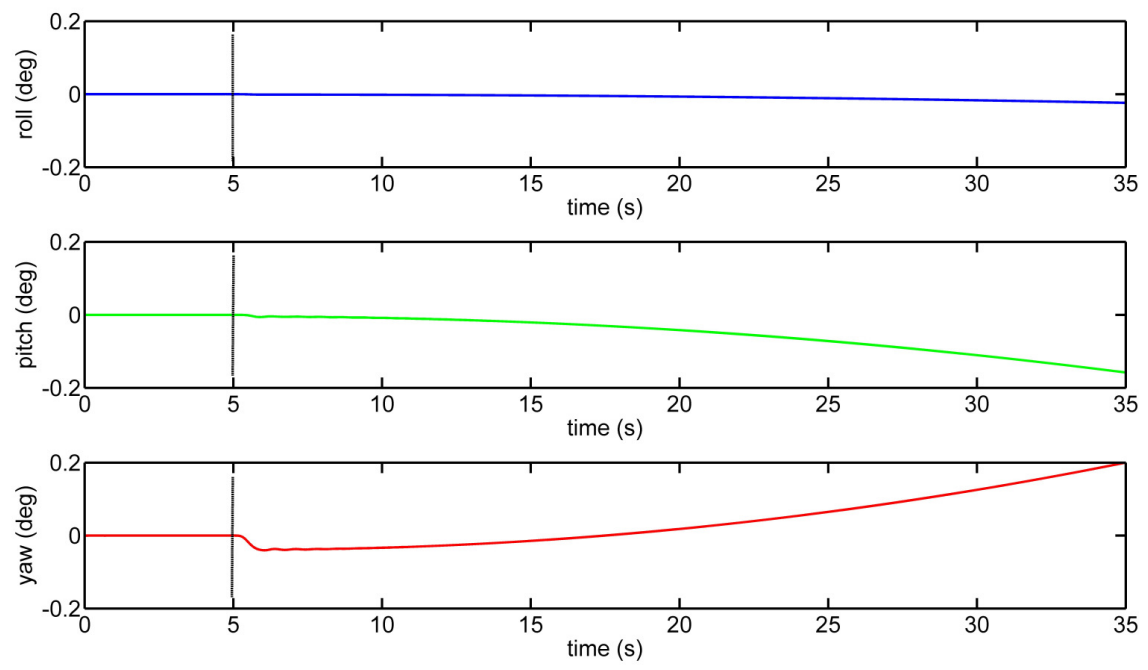

Fig. 17 Euler angles representing the system's attitude during the propulsive phase (aligned case)

\section{Conclusion}

The paper discusses the issue of the alignment between the center of mass and the thrust direction arising after the grasping phases of an on-orbit servicing (or debris removal) mission, in preparation for further maneuvers.

The problem of the estimation of the a-priori unknown moments of inertia and the center of mass position of the resulting multibody has been first analyzed. Measurements of the angular rates produced by a sequence of perfectly known thrust pulses are considered as available data. The extended Kalman filter has been identified as suitable estimation tool, and numerical simulations show that this method produces reliable results. A specific aspect of the estimation process is that only the components of the center of mass position vector normal to the thrust direction can be evaluated. The necessity of having different thrust directions is therefore mandatory, in order to reconstruct completely the mass distribution on the system. The estimation of the moments of inertia is more challenging, with the Kalman filter converging slowly with respect to the case of center of mass.

A numerical robustness analysis has been also numerically performed in order to investigate the sensitivity of the filter in presence of greater misalignment with respect to the nominal case. The obtained results show that the estimation is successful when the error on the initial guess is not too large.

The correct knowledge of the position of the center of mass and of the moment of inertia of the system is mandatory for the success of the following re-alignment maneuver. This maneuver can be performed by a reconfiguration of the links of the manipulator that operated the grasping, while the end effectors of the same arms do not lose their clamping point on the target. The actions to be commanded to the motors of the robotic arms are computed via the Jacobian transpose control approach. Numerical simulations proofing the suitability of the proposed technique are presented.

As a result of this method, every following maneuver involving a thrust and operated by the servicing platform can be correctly exploited, neglecting any risk coming from the misalignment, even if the thrusting pulse - as in the case of a debris removal mission aiming to destroy the target by means of a forced re-entry - should be extremely large.

\section{Appendix}

The derivation of the Jacobian matrix by means of the Denavit-Hartenberg approach is a procedure commonly used in terrestrial robotic systems ${ }^{15}$. The derivation starts from the definition of the reference frames associated to the link of the manipulator by following the Denavit-Hartenberg conventions ${ }^{16}$ and then uses the homogeneous matrix for representing the mutual position and attitude between two neighbor links as follows: 


$$
\mathbf{M}_{i-1, i}=\left[\begin{array}{cc}
\mathbf{R}_{i-1, i} & \vec{D}_{i-1, i} \\
0 & 1
\end{array}\right]=\left[\begin{array}{cccc}
c_{\vartheta_{i}} & -s_{\vartheta_{i}} c_{\alpha_{i}} & s_{\vartheta_{i}} s_{\alpha_{i}} & a_{i} c_{\vartheta_{i}} \\
s_{\vartheta_{i}} & c_{\vartheta_{i}} c_{\alpha_{i}} & -c_{\vartheta_{i}} s_{\alpha_{i}} & a_{i} s_{\vartheta_{i}} \\
0 & s_{\alpha_{i}} & c_{\alpha_{i}} & d_{i} \\
0 & 0 & 0 & 1
\end{array}\right]
$$

where $a_{i}, d_{i}, \alpha_{i}$ and $\vartheta_{i}$ (already reported in Table 2 for the case under investigation) are the length, the offset, the torsion angle and the joint rotation angle, respectively. The direction of the axis of the revolute joint associated to the motion of the $i$-th link with respect to the previous one can be represented by the matrix:

$$
\mathbf{L}_{i-1, i}=\left[\begin{array}{cccc}
0 & -1 & 0 & 0 \\
1 & 0 & 0 & 0 \\
0 & 0 & 0 & 0 \\
0 & 0 & 0 & 0
\end{array}\right]
$$

It is possible to report this direction to the reference frame associated to the base of the manipulator (0) by applying the following rule:

$$
{ }^{0} \mathbf{L}_{i-1, i}=\left(\mathbf{M}_{0,1} \mathbf{M}_{1,2} \ldots \mathbf{M}_{\mathrm{i}-2, \mathrm{i}-1}\right) \mathbf{L}_{i-1, i}\left(\mathbf{M}_{0,1} \mathbf{M}_{1,2} \ldots \mathbf{M}_{\mathrm{i}-2, \mathrm{i}-1}\right)^{-1}=\mathbf{M}_{0, i-1}{ }^{0} \mathbf{L}_{i-1, i} \mathbf{M}_{0, i-1}^{-1}
$$

where the resulting position matrix $\mathbf{M}_{0, i-1}$ is obtained as the product of the matrices relevant to the sequence of the links from the base 0 to the $i$-th body of the kinematic chain. The resulting matrix ${ }^{0} \mathbf{L}_{i-1, i}$ has the following structure:

$$
{ }^{0} \mathbf{L}_{i-1, i}=\left[\begin{array}{cccc}
0 & -{ }^{0} L_{i-1, i}^{z} & { }^{0} L_{i-1, i}^{y} & { }^{0} T_{i-1, i}^{x} \\
{ }^{0} L_{i-1, i}^{z} & 0 & -{ }^{0} L_{i-1, i}^{x} & { }^{0} T_{i-1, i}^{y} \\
-{ }^{0} L_{i-1, i}^{y} & { }^{0} L_{i-1, i}^{x} & 0 & { }^{0} T_{i-1, i}^{y} \\
0 & 0 & 0 & 0
\end{array}\right]
$$

where ${ }^{0} L_{i-1, i}^{x},{ }^{0} L_{i-1, i}^{y}$ and ${ }^{0} L_{i-1, i}^{z}$ are the components of the unit vector representing the rotation of the $i$-th link with respect to the 0 -th reference frame, due to the motion of the joint between the $(i-1)$-th and $i$-th link. In the same way ${ }^{0} T_{i-1, i}^{x},{ }^{0} T_{i-1, i}^{y}$ and ${ }^{0} T_{i-1, i}^{z}$ are the components of the unit vector representing the translation of the $i$-th link with respect to the 0 -th reference frame, due to the motion of the joint between the $(i-1)$-th and $i$-th link.

The Jacobian matrix, representing the motion of the end effector with respect to the point where is attached the manipulator, is built by selecting the translation unit vector components $\left({ }^{0} T_{i-1, i}^{x},{ }^{0} T_{i-1, i}^{y}\right.$ and $\left.{ }^{0} T_{i-1, i}^{z}\right)$ for each joint of the manipulator and collecting them as follows:

$$
\mathbf{J}=\left[\begin{array}{cccc}
{ }^{0} T_{0,1}^{x} & { }^{0} T_{1,2}^{x} & \cdots & { }^{0} T_{N-1, \mathrm{~N}}^{x} \\
{ }^{0} T_{0,1}^{y} & { }^{0} T_{1,2}^{x} & \cdots & { }^{0} T_{N-1, \mathrm{~N}}^{x} \\
{ }^{0} T_{0,1}^{z} & { }^{0} T_{1,2}^{x} & \cdots & { }^{0} T_{N-1, \mathrm{~N}}^{x}
\end{array}\right]
$$

This matrix allows to formulate the relationship between the end effector velocity and the joints' coordinates rates $\dot{Q}$ by means of:

$$
{ }^{0} \vec{V}_{e e}=\mathbf{J} \dot{\mathrm{Q}}
$$

which can be used for the Jacobian transpose control approach as shown in previous Eq.(25). 


\section{References}

${ }^{1}$ Bonnal, C., Ruault, J.M., Desjean, M.C., , "Active debris removal: Recent progress and current trends", Acta Astronautica, Vol. 85, Apr.-May 2013, pp. 51-60.

${ }^{2}$ Castronuovo, M.M., "Active space debris removal-A preliminary mission analysis and design", Acta Astronautica, Vol. 69, No. 9-10, Nov.-Dec. 2011, pp. 848-859

${ }^{3}$ Palmerini, G.B., Sabatini, M., Gasbarri, P., Monti, R., Felicetti, L., "Design of debris removal missions performed by robotic graspers”, IAC-12-C2.2.11, 63rd International Astronautical Congress, Naples, 2012.

${ }^{4}$ Toglia, C., Palmerini, G.B., Gasbarri, P., "Basic Aspects in Designing Space Grasper Missions", IAC-07-D1.1.07, 58th International Astronautical Congress, Hyderabad, 2007

${ }^{5}$ Xu, W., Liang, B., Xu., Y., "Survey of Modeling, Planning, and Ground Verification of Space Robotic Systems", Acta Astronautica, 2011, Vol.68, No.11-12, pp. 1629-1649

${ }^{6}$ Bergmann, E., Walker, B., Levy, D., "Mass property estimation for control of asymmetrical satellites", Guidance, Navigation and Control Conference, 1985, AIAA 1985-1857

${ }^{7}$ Wilson, E., Sutter, D., Mah, R., "Motion-Based Mass- and Thruster-Property Identification for Thruster-Controlled Spacecraft”, Infotech@Aerospace, 26-29 Sep. 2005, Arlington, Virginia, AIAA 2005-6907

${ }^{8}$ Tanygin, S., Williams, T., "Mass Property Estimation Using Coasting Maneuvers", Journal of Guidance, Control, and Dynamics, 1997, Vol.20, No. 4, pp. 625-632

${ }^{9}$ Norman, M.C., Peck, M.A., O’Shaughnessy, D.J., "In-Orbit Estimation of Inertia and Momentum Actuator Alignment Parameters," Journal of Guidance, Control, and Dynamics, 2011, Vol. 34 No.6, pp. 1798-1814

${ }^{10}$ Van Dyke, M. C., Schwartz, J. L., Hall, C. D., "Unscented Kalman Filtering for Spacecraft Attitude State and Parameter Estimation," AAS/AIAA space flight mechanics meeting, Feb. 8-12 2004, Maui, Hawaii, AAS-04-115, Advances in Astronautical Sciences, 119, pp. 217-228, 2004

${ }^{11}$ Sekhavat, P., Karpenko, M., Ross, I., "UKF-Based Spacecraft Parameter Estimation Using Optimal Excitation", AIAA Guidance, Navigation, and Control Conference, 10-13 Aug. 2009, Chicago, Illinois, AIAA 2009-5786

${ }^{12}$ Zarchan, P., Musoff, H., "Fundamentals of Kalman Filtering - A Practical Approach", Progress in Astronautics and Aeronautics Series, Vol.190, AIAA Publ., 2009.

${ }^{13}$ Montenbruck, O., Gill, E., "Satellite Orbits: Models, Methods and Applications”, Springer, 2011.

${ }^{14}$ Jazar, R.N., "Advanced Dynamics: Rigid Body, Multibody, and Aerospace Applications”, John Wiley \& Sons Inc., 2011.

${ }^{15}$ Siciliano, B., Schiavicco, L., Villani, L., Oriolo, G., "Robotics: modelling, planning and control", Springer, 2009.

${ }^{16}$ Denavit, J., Hartenberg, R.S., "A Kinematics Notation for Lower Pair Mechanisms based on Matrices", Transactions ASME Journal Applied Mechanics, Vol.22, pp.215-221, 1955 